\title{
A GENERAL CLASS OF FREE BOUNDARY PROBLEMS FOR FULLY NONLINEAR PARABOLIC EQUATIONS
}

\author{
ALESSIO FIGALLI AND HENRIK SHAHGHOLIAN
}

Abstract. In this paper we consider the fully nonlinear parabolic free boundary problem

$$
\begin{cases}F\left(D^{2} u\right)-\partial_{t} u=1 & \text { a.e. in } Q_{1} \cap \Omega \\ \left|D^{2} u\right|+\left|\partial_{t} u\right| \leq K & \text { a.e. in } Q_{1} \backslash \Omega\end{cases}
$$

where $K>0$ is a positive constant, and $\Omega$ is an (unknown) open set.

Our main result is the optimal regularity for solutions to this problem: namely, we prove that $W_{x}^{2, n} \cap W_{t}^{1, n}$ solutions are locally $C_{x}^{1,1} \cap C_{t}^{0,1}$ inside $Q_{1}$. A key starting point for this result is a new BMO-type estimate which extends to the parabolic setting the main result in [4].

Once optimal regularity for $u$ is obtained, we also show regularity for the free boundary $\partial \Omega \cap Q_{1}$ under the extra condition that $\Omega \supset\{u \neq 0\}$, and a uniform thickness assumption on the coincidence set $\{u=0\}$,

\section{INTRODUCTION AND MAIN RESUlT}

1.1. Setting of the problem. In this paper we will use $Q_{r}(X):=B_{r}(x) \times(t-r, t) \subset \mathbb{R}^{n} \times \mathbb{R}$ to denote the parabolic ball of radius $r$ centered at a point $X=(x, t) \in \mathbb{R}^{n+1}$, and we will use the notation $Q_{r}=Q_{r}(0)$.

Our starting point will be a $W_{x}^{2, n}\left(Q_{1}\right) \cap W_{t}^{1, n}\left(Q_{1}\right)$ function $u: Q_{1} \rightarrow \mathbb{R}$ satisfying

$$
\begin{cases}\mathcal{H}(u)=1 & \text { a.e. in } Q_{1} \cap \Omega \\ \left|\tilde{D}^{2} u\right| \leq K & \text { a.e. in } Q_{1} \backslash \Omega\end{cases}
$$

where $\tilde{D}^{2} u=\left(D_{x}^{2} u, D_{t} u\right) \in \mathbb{R}^{n^{2}+1}, \mathcal{H}(u):=F\left(D^{2} u\right)-\partial_{t} u, K>0$, and $\Omega \subset \mathbb{R}^{n+1}$ is some unknown open set. Since, by assumption, $\tilde{D}^{2} u$ is bounded in the complement of $\Omega$, we see that $\mathcal{H}(u)$ is bounded inside the whole $Q_{1}$ and $u$ is a so-called "strong $L^{n}$ solution" to a fully nonlinear parabolic equation with bounded right hand side [7]. We refer to [12, 7] as basic references to parabolic fully nonlinear equations and viscosity methods.

The above free boundary problem has a very general form and encompasses several other free boundaries of obstacle type. In the elliptic case, it has been recently studied by the authors in [10. We also refer to several other articles concerning similar type of problems: For elliptic case see [5], [1], and for parabolic case see [6], [2]. One may find applications and relevant discussions about these kinds of problems in these articles.

Since most of the result follow the same line of arguments (sometimes with obvious modifications) as that of its elliptic counterpart done in [10], here we have chosen not to enter into the details of the proof as they can be worked out in a similar way as in the elliptic case. Instead, we shall give the outline of the proofs and point out all the necessary changes. For the reader

A. Figalli was partially supported by NSF grant DMS-1262411. 
unfamiliar with these techniques, we suggest first to read [10].

Going back to our problem, we observe that, if $u \in W_{x}^{2, n} \cap W_{t}^{1, n}$, then $\tilde{D}^{2} u=0$ a.e. inside $\{u=0\}$, and $D^{2} u=0$ a.e. inside $\{\nabla u=0\}$. In particular we easily deduce that (1.1) includes, as special cases, both $\mathcal{H}(u)=\chi_{\{u \neq 0\}}$ and $\mathcal{H}(u)=\chi_{\{\nabla u \neq 0\}}$.

We assume that:

(H0) $F(0)=0$.

(H1) $F$ is uniformly elliptic with ellipticity constants $0<\lambda_{0} \leq \lambda_{1}<\infty$, that is,

$$
\mathscr{P}^{-}\left(P_{1}-P_{2}\right) \leq F\left(P_{1}\right)-F\left(P_{2}\right) \leq \mathscr{P}^{+}\left(P_{1}-P_{2}\right)
$$

for any $P_{1}, P_{2}$ symmetric, where $\mathscr{P}^{-}$and $\mathscr{P}^{+}$are the extremal Pucci operators:

$$
\mathscr{P}^{-}(M):=\inf _{\lambda_{0} \operatorname{Id} \leq N \leq \lambda_{1} \operatorname{Id}} \operatorname{trace}(N M), \quad \mathscr{P}^{+}(M):=\sup _{\lambda_{0} \operatorname{Id} \leq N \leq \lambda_{1} \operatorname{Id}} \operatorname{trace}(N M) .
$$

(H2) $F$ is either convex or concave.

Under assumptions (H0)-(H2) above, strong $L^{n}$ solutions are also viscosity solutions [5], and hence regularity results for parabolic fully nonlinear equations [12, 13] show that $u \in W_{x}^{2, p}\left(Q_{\rho}\right) \cap$ $W_{t}^{1, p}\left(Q_{\rho}\right)$ for all $\rho \in(0,1)$ and $p<\infty$.

Our first result concern the optimal $C_{x}^{1,1} \cap C_{t}^{0,1}$-regularity for $u$. Once this will be done, we will be able to study the regularity of the free boundary.

1.2. Main results. Our first result concerns the optimal regularity of solutions to (1.1):

Theorem 1.1. (Interior $C_{x}^{1,1} \cap C_{t}^{0,1}$ regularity) Let $u: Q_{1} \rightarrow \mathbb{R}$ be $a W_{x}^{2, n} \cap W_{t}^{1, n}$ solution of (1.1), and assume that $F$ satisfies (H0)-(H2). Then there exists a constant $\bar{C}=\bar{C}\left(n, \lambda_{0}, \lambda_{1},\|u\|_{\infty}\right)>0$ such that

$$
\left|\tilde{D}^{2} u\right| \leq \bar{C}, \quad \text { in } Q_{1 / 2}
$$

To state our result on the regularity of the free boundary, we need to introduce the concept of minimal diameter: Set $\Lambda:=Q_{1} \backslash \Omega$, and for any set $E \in \mathbb{R}^{n}$ let $\operatorname{MD}(E)$ denote the smallest possible distance between two parallel hyperplanes containing $E$. Then, given a point $X^{0}=$ $\left(x^{0}, t^{0}\right) \in \mathbb{R}^{n+1}$, we define

$$
\delta_{r}\left(u, X^{0}\right):=\inf _{t \in\left[t_{0}-r^{2}, t_{0}+r^{2}\right]} \frac{\operatorname{MD}\left(\Lambda \cap\left(B_{r}\left(x^{0}\right) \times\{t\}\right)\right)}{r} .
$$

In other words, $\delta_{r}\left(u, X^{0}\right)$ measures the thickness of the complement of $\Omega$ at all time levels $t \in\left(t^{0}-r^{2}, t^{0}+r^{2}\right)$, around the point $x^{0}$. Notice that $\delta_{r}$ depends on $u$ since $\Omega$ does. In particular, we observe that if $u$ solves (1.1) for some set $\Omega$, then $u_{r}(y, \tau):=u\left(x+r y, t+r^{2} \tau\right) / r^{2}$ solves (1.1) with

$$
\Omega_{r}:=\left\{(y, \tau):\left(x+r y, t+r^{2} \tau\right) \in \Omega\right\}
$$

in place of $\Omega$, and $\delta_{r}$ enjoys the scaling property $\delta_{1}\left(u_{r}, 0\right)=\delta_{r}(u, X), X=(x, t)$.

Our result provides regularity for the free boundary under a uniform thickness condition. As a corollary of our result, we deduce that Lipschitz free boundaries are $C^{1}$, and hence smooth [8]. 
Theorem 1.2. (Free boundary regularity) Let $u: Q_{1} \rightarrow \mathbb{R}$ be $a W_{x}^{2, n} \cap W_{t}^{1, n}$ solution of (1.1). Assume that $F$ is convex and satisfies (HO)-(H1), and that $\Omega \supset\{u \neq 0\}$. Suppose further that there exists $\varepsilon>0$ such that

$$
\delta_{r}(u, z)>\varepsilon \quad \forall r<1 / 4, z \in \partial \Omega \cap Q_{r}(0) .
$$

Then $\partial \Omega \cap Q_{r_{0}}(0)$ is a $C^{1}$-graph in space-time, where $r_{0}$ depends only on $\varepsilon$ and the data.

The paper is organized as follows:

In Section 2 we prove Theorem 1.1. Then in Section 3 we investigate the non-degeneracy of solutions, and classify global solutions under a suitable thickness assumption. In Section 4 we show directional monotonicity for local solutions which gives Lipschitz (and then $C^{1}$ ) regularity for the free boundary, as shown in Section 5 .

\section{Proof of Theorem 1.1}

The proof of this theorem follows the same line of ideas as its elliptic counterpart [10]. First one starts from a BMO-type estimate on $D^{2} u$, and then one shows a dichotomy that either $u$ has quadratic growth away from a free boundary point $X^{0}$, or the density of the set $\Lambda$ at $X^{0}$ vanishes fast enough to assure the quadratic bound.

In [10] the following result was a consequence of the BMO-type estimate proved in [4]. Since we could not find a reference for this estimate in the parabolic case, we prove this result in the appendix. We notice that our proof is much simpler than the one in [4] and actually gives a new proof of the results there (see Remark 6.3).

With no loss of generality, we will carry out the proof at the origin, by letting $X^{0}=(0,0)$.

Lemma 2.1. There exist a constant $C=C\left(n, \lambda_{0}, \lambda_{1},\|u\|_{\infty}\right)$, and a family of second order parabolic polynomial $\left\{P_{r}\right\}_{r \in(0,1)}$ solving $\mathcal{H}\left(P_{r}\right)=0$, such that

$$
\sup _{Q_{r}(0)}\left|u-P_{r}\right| \leq C r^{2}, \quad \forall r \in(0,1) .
$$

Consequently

$$
\sup _{Q_{r}(0)}|u| \leq\left(C r^{2}+\left|P_{r}\right|\right), \quad \forall r \in(0,1) .
$$

The first statement in the Lemma is proven in Appendix (see (6.1) and Lemma 6.2 there), while the second estimate is a straightforward consequence of the first one. It should be remarked that these polynomials $P_{r}$ need not to be unique.

Define

$$
A_{r}:=\left\{(x, t):\left(r x, r^{2} t\right) \in Q_{r} \backslash \Omega\right\} \subset Q_{1} \quad \forall r<1 / 4 .
$$

We shall prove that if $\left|P_{r}\right|$ is sufficiently large then the measure of $A_{r}$ has to decay geometrically.

Proposition 2.2. Set $\tilde{P}_{r}:=\tilde{D}^{2} P_{r}$. There exists $M>0$ universal such that, for any $r \in(0,1 / 8)$, if $\left|\tilde{P}_{r}\right| \geq M$ then

$$
\left|A_{r / 2}\right| \leq \frac{\left|A_{r}\right|}{2^{n+1}}
$$

The proof of the proposition follows the same lines of ideas as that of [10, Proposition 2.4]. However, since the changes are not completely straightforward, for the reader's convenience we present the proof here. 
Proof. Set $u_{r}(y, t):=u\left(r y, r^{2}\right) / r^{2}, \mathcal{H}_{r}(v):=F\left(D^{2} P_{r}+D^{2} v\right)-\partial_{t} P_{r}-\partial_{t} v$, and let

$$
u_{r}(y, t)=P_{r}(y, t)+v_{r}(y, t)+w_{r}(y, t),
$$

where $v_{r}$ is defined as the solution of

$$
\begin{cases}\mathcal{H}_{r}\left(P_{r}+v_{r}\right)-1=0 & \text { in } Q_{1}, \\ v_{r}(y, t)=u_{r}(y, t)-P_{r}(y, t) & \text { on } \partial_{p} Q_{1},\end{cases}
$$

where $\partial_{p} Q_{1}$ denotes the parabolic boundary of $Q_{1}$, and by definition $w_{r}:=u_{r}-P_{r}-v_{r}$.

Set $f_{r}:=\mathcal{H}\left(D^{2} u_{r}\right) \in L^{\infty}\left(B_{1}\right)$ (recall that $\left|\tilde{D}^{2} u_{r}\right| \leq K$ a.e. inside $\left.A_{r}\right)$. Notice that, since $f_{r}=1$ outside $A_{r}$,

so it follows by (H1) that $w_{r}$ solves

$$
\mathcal{H}\left(u_{r}\right)-\mathcal{H}\left(P_{r}+v_{r}\right)=\left(f_{r}-1\right) \chi_{A_{r}},
$$

$$
\begin{cases}\mathscr{P}^{-}\left(D^{2} w_{r}\right)-\partial_{t} w_{r} \leq\left(f_{r}-1\right) \chi_{A_{r}} \leq \mathscr{P}^{+}\left(D^{2} w_{r}\right)-\partial w_{r} & \text { in } Q_{1}, \\ w_{r}=0 & \text { on } \partial_{p} Q_{1} .\end{cases}
$$

Hence, we can apply the ABP estimate [12, Theorem 3.14] to deduce that

$$
\sup _{Q_{1}}\left|w_{r}\right| \leq C\left\|\chi_{A_{r}}\right\|_{L^{n+1}\left(Q_{1}\right)}=C\left|A_{r}\right|^{1 /(n+1)} .
$$

Also, since $\mathcal{H}\left(P_{r}\right)=0$ and $v_{r}$ is universally bounded on $\partial_{p} Q_{1}$ (see (2.1) and (2.5)), by the parabolic Evans-Krylov's theorem [9] applied to (2.5) we have

$$
\left\|\tilde{D}^{2} v_{r}\right\|_{C^{0, \alpha}\left(Q_{3 / 4}\right)} \leq C .
$$

This implies that $w_{r}$ solves the fully nonlinear equation with Hölder coefficients

$G\left(D^{2} w_{r}, X\right)-\partial_{t} w_{r}-\partial_{t}\left(v_{r}+P_{r}\right)=\left(f_{r}-1\right) \chi_{A_{r}} \quad$ in $Q_{3 / 4}, \quad G(M, X):=F\left(D^{2} P_{r}+D^{2} v_{r}(x)+M\right)-1$.

Since $G(0, X)=0$, we can apply [12, Theorem 5.6] with $p=n+2$ and (2.7) to obtain

$$
\int_{Q_{1 / 2}}\left|D^{2} w_{r}\right|^{n+2} \leq C\left(\left\|w_{r}\right\|_{L^{\infty}\left(Q_{3 / 4}\right)}+\left\|\chi_{A_{r}}\right\|_{L^{2 n}\left(Q_{3 / 4}\right)}\right)^{n+2} \leq C\left|A_{r}\right|
$$

(recall that $\left|A_{r}\right| \leq\left|Q_{1}\right|$ ).

We are now ready to conclude the proof: since $\left|\tilde{D}^{2} u_{r}\right| \leq K$ a.e. inside $A_{r}$ (by (1.1)), recalling (2.4) we have

$$
\int_{A_{r} \cap Q_{1 / 2}}\left|\tilde{D}^{2} v_{r}+\tilde{D}^{2} w_{r}+\tilde{P}_{r}\right|^{n+2}=\int_{A_{r} \cap Q_{1 / 2}}\left|\tilde{D}^{2} u_{r}\right|^{n+2} \leq K^{n+2}\left|A_{r}\right| .
$$

Therefore, by (2.8) and (2.9),

$$
\begin{aligned}
\left|A_{r} \cap Q_{1 / 2}\right|\left|\tilde{P}_{r}\right|^{n+2} & =\int_{A_{r} \cap Q_{1 / 2}}\left|\tilde{P}_{r}\right|^{n+2} \\
& \leq 3^{2 n}\left(\int_{A_{r} \cap Q_{1 / 2}}\left|\tilde{D}^{2} v_{r}\right|^{n+2}+\int_{A_{r} \cap Q_{1 / 2}}\left|\tilde{D}^{2} w_{r}\right|^{n+2}+K^{n+2}\left|A_{r}\right|\right) \\
& \leq 3^{n+2}\left(\left|A_{r} \cap Q_{1 / 2}\right|\left\|\tilde{D}^{2} v_{r}\right\|_{L^{\infty}\left(Q_{1 / 2}\right)}+\int_{Q_{1 / 2}}\left|\tilde{D}^{2} w_{r}\right|^{n+2}+K^{n+2}\left|A_{r}\right|\right) \\
& \leq C\left|A_{r} \cap Q_{1 / 2}\right|+C\left|A_{r}\right| .
\end{aligned}
$$


Hence, if $\left|\tilde{P}_{r}\right|$ is sufficiently large we obtain

$$
\left|A_{r} \cap Q_{1 / 2}(0)\right|\left|\tilde{P}_{r}\right|^{n+2} \leq C\left|A_{r}\right| \leq \frac{1}{4^{n+1}}\left|\tilde{P}_{r}\right|^{n+2}\left|A_{r}\right| .
$$

Since $\left|A_{r / 2}\right|=2^{n+1}\left|A_{r} \cap Q_{1 / 2}(0)\right|$, this gives the desired result.

2.1. Proof of Theorem 1.1. Taking $M>0$ as in Proposition 2.2, we have that one of the following hold:

(i) $\liminf _{k \rightarrow \infty}\left|P_{2^{-k}}\right| \leq 3 M$,

(ii) $\liminf _{k \rightarrow \infty}\left|P_{2^{-k}}\right| \geq 3 M$.

Then, one consider the two case separately and, arguing exactly as in the proof of Theorem 1.2 in [10] one obtains the desired result. (We notice that the reference [3, Theorem 3] in that proof is to be replaced by [13, Theorem 1.1].)

\section{Non-DEgeneracy And global solutions}

3.1. Local non-degeneracy. As shown in [10, Section 3] non-degeneracy fails in general for the elliptic case, and surely for our problem as well. Nevertheless, the non-degeneracy does hold for the case $\Omega \supset\{\nabla u \neq 0\}$, see [10, Lemma 3.1]. We now show that this non-degeneracy result still holds in the parabolic case:

Lemma 3.1. Let $u: Q_{1} \rightarrow \mathbb{R}$ be a $W_{x}^{2, n} \cap W_{t}^{1, n}$ solution of (1.1), assume that $F$ satisfies (H0)-(H2), and that $\Omega \supset\{\nabla u \neq 0\}$. Then, for any $X^{0}=\left(x^{0}, t^{0}\right) \in \bar{\Omega} \cap Q_{1 / 2}$,

$$
\max _{\partial_{p} Q_{r}\left(X^{0}\right)} u \geq u\left(X^{0}\right)+\frac{r^{2}}{2 n \lambda_{1}+1} \quad \forall r \in(0,1 / 4) .
$$

Proof. For

$$
v(x):=u(x)-\frac{\left|x-x^{0}\right|^{2}-\left(t-t^{0}\right)}{2 n \lambda_{1}+1},
$$

one readily verifies that $\mathcal{H}(v) \geq 0$ in $Q_{r}\left(X^{0}\right)$. Then, by the very same argument as in the proof of [10, Lemma 3.1] we deduce that

$$
\max _{\partial_{p} Q_{r}\left(X^{0}\right)} v=\sup _{Q_{r}\left(X^{0}\right)} v
$$

and the result follows easily.

3.2. Classification of global solutions. As already discussed in the previous section, to have non-degeneracy of solutions we need to assume that $\Omega \supset\{\nabla u \neq 0\}$. In the elliptic case this assumption is also sufficient to classify global solutions with a "thick free boundary" (see [10, Proposition 3.2]). However, in the parabolic case the situation is much more complicated: indeed, while global solutions of the elliptic problem with "thick free boundary" are convex and onedimensional, in the parabolic case we have non-convex solutions. For instance the function

$$
u(x)= \begin{cases}-2 t-x_{1}^{2} / 2 & \text { if } x_{1}>0, \\ -2 t & \text { if } x_{1} \leq 0,\end{cases}
$$

is a global solution to the problem $\Delta u-\partial_{t} u=\chi_{\{\nabla u \neq 0\}}$.

In order to avoid these examples, here we shall only consider the case $\Omega \supset\{u \neq 0\}$. 
Since we will use minimal diameter to measure sets, we need some facts about their stability properties: Let us first recall the definition for $\delta_{r}\left(u, X^{0}\right)$ for $X^{0}=\left(x^{0}, t^{0}\right)$ :

$$
\delta_{r}\left(u, X^{0}\right):=\inf _{t \in\left[t_{0}-r^{2}, t_{0}+r^{2}\right]} \frac{\operatorname{MD}\left(\Lambda \cap\left(B_{r}\left(x^{0}\right) \times\{t\}\right)\right)}{r}, \quad \Lambda:=Q_{1} \backslash \Omega .
$$

We remark that, for polynomial global solutions $P_{2}=\sum_{j} a_{j} x_{j}^{2}+b t$ (with $A=\operatorname{diag}\left(a_{j}\right)$, and $b$ such that $F(A)-b=1$ ), one has

$$
\delta_{r}\left(P_{2}, 0\right)=0 .
$$

Let us also recall the standard scaling and stability estimate

$$
\delta_{r}(u, X)=\delta_{1}\left(u_{r}, 0\right), \quad \limsup _{r \rightarrow 0} \delta_{r}\left(u, X^{0}\right) \leq \delta_{1}\left(u_{0}, 0\right)
$$

whenever $u_{r}(y, \tau)=u\left(x+r y, t+r^{2} \tau\right) / r^{2}$ converges uniformly to some function $u_{0}$.

In the next proposition we classify global solution with a "thick free boundary". We notice that assumption (3.3) below allows us to exclude the family of global solutions $u_{\sigma}(t, x)=-(t-\sigma)_{+}$, $\sigma \in \mathbb{R}$.

Proposition 3.2. Let $u: \mathbb{R}^{n+1} \rightarrow \mathbb{R}$ be a $W^{2, n}$ solution of (1.1) on the whole $\mathbb{R}^{n+1}$, assume that $F$ is convex and satisfies (H0)-(H1), and that $\Omega \supset\{u \neq 0\}$. Furthermore, assume that there exists $\epsilon_{0}>0$ such that

$$
\delta_{r}\left(u, X^{0}\right) \geq \epsilon_{0} \quad \forall r>0, \forall X^{0} \in \partial \Omega .
$$

Then $u$ is time-independent. In particular, by the elliptic case [10, Proposition 3.2], $u$ is a halfspace solution, i.e., up to a rotation, $u(x)=\gamma\left[\left(x_{1}\right)_{+}\right]^{2} / 2$, where $\gamma \in\left(1 / \lambda_{1}, 1 / \lambda_{0}\right)$ is such that $F\left(\gamma e_{1} \otimes e_{1}\right)=1$.

Proof. Let $m:=\sup _{\mathbb{R}^{n+1}} \partial_{t} u$ (notice that $m$ is finite by Theorem 1.1) and consider a sequence $m_{j}=\partial_{t} u\left(X^{j}\right)$ such that $m_{j} \rightarrow m$.

We now perform the scaling

$$
u_{j}(x, t):=\frac{u\left(d_{j} x+x^{j}, d_{j}^{2} t+t^{j}\right)}{d_{j}^{2}}
$$

where $X^{j}=\left(x^{j}, t^{j}\right)$ and $d_{j}:=\operatorname{dist}\left(X^{j}, \partial \Omega\right)$.

The functions $u_{j}$ still satisfy (1.1). Also, since $u=0$ on $\partial \Omega$ it follows by the $C_{x}^{1,1} \cap C_{t}^{0,1}$ regularity of $u$ that $u_{j}$ are uniformly bounded, hence, up to subsequences, they converge to another global solution $u_{\infty}$ which satisfies $\partial_{t} u_{\infty}(0)=m$. By (3.2) and the assumption (3.3) we obtain

$$
\delta_{r}\left(u_{\infty}, X^{0}\right) \geq \epsilon_{0} \quad \forall r>0, \forall X^{0} \in \partial \Omega_{\infty},
$$

where $\Omega_{\infty}$ is the limit, as $j \rightarrow \infty$, of the family of open sets

$$
\Omega_{j}:=\left\{(x, t):\left(d_{j} x+x^{j}, d_{j}^{2} t+t^{j}\right) \in \Omega\right\} .
$$

Let us observe that, by the condition $\Omega \supset\{u \neq 0\}$ we get $u_{\infty}(t, x)=0$ on $\partial \Omega_{\infty}$.

In addition $\partial_{t} u_{\infty}$ is a solution of the uniformly parabolic linear operator $F_{i j}\left(D^{2} u_{\infty}\right) \partial_{i j}-\partial_{t}$ inside $\Omega_{\infty}$. Hence, since $\partial_{t} u_{\infty} \leq m$ and $\partial_{t} u_{\infty}(0)=m$, by the strong maximum principle we deduce that $\partial_{t} u_{\infty}$ is constant inside the connected component of $\Omega_{\infty}$ containing 0 (call it $\Omega_{0}$ ).

Therefore, integrating $u_{\infty}$ in the direction $t$ gives

$$
u_{\infty}(t, x)=m t+U(x) \quad \text { inside } \Omega_{0}, \quad u_{\infty}=0 \quad \text { on } \partial \Omega_{0} .
$$


Thanks to the assumption (3.3) it is easy to check that $\partial \Omega_{0}$ cannot be contained into hyperplanes of the form $\{t=\sigma\}$ for $\sigma \in \mathbb{R}$, hence (3.5) is possible only if $m=0$. Hence we have proved that $\sup _{\mathbb{R}^{n+1}} \partial_{t} u=0$.

By a completely symmetric argument we obtain $\inf _{\mathbb{R}^{n+1}} \partial_{t} u=0$. Thus $\partial_{t} u=0$, which implies that $u$ is time-independent and therefore, by [10, Proposition 3.2], up to a rotation $u$ is of the form $u(x)=\gamma\left[\left(x_{1}\right)_{+}\right]^{2} / 2+c \gamma \in\left(1 / \lambda_{1}, 1 / \lambda_{0}\right)$ is such that $F\left(\gamma e_{1} \otimes e_{1}\right)=1$ and $c \in \mathbb{R}$. Since $\Omega \supset\{u \neq 0\}$ we see that $c=0$, which proves the result.

\section{LoCAL SOlutions AND DiRECTIONAL MONOTONICITY}

In this section we shall prove a directional monotonicity for solutions to our equations. In the next section we will use Lemma 4.2 below to show that, if $u$ is close enough to a half-space solution $\gamma\left[\left(x_{1}\right)_{+}\right]^{2}$ in a ball $B_{r}$, then for any $e=\left(e_{x}, e_{t}\right) \in \mathbb{S}^{n}$ with $e \cdot\left(e_{1}, 0\right) \geq s>0$ we have $C_{0} \partial_{e} u-u \geq 0$ inside $B_{r / 2}$.

Lemma 4.1. Let $u: Q_{1} \rightarrow \mathbb{R}$ be $a W_{x}^{2, n} \cap W_{t}^{1, n}$ solution of (1.1) with $\Omega \supset\{u \neq 0\}$. Then, under the conditions of Theorem 1.2 we have

$$
\lim _{\Omega \ni X \rightarrow \partial \Omega} \partial_{t} u(X)=0 .
$$

Proof. The proof of this lemma follows easily by a contradictory argument, along with scaling and blow-up. Indeed, given a sequence $X^{j} \rightarrow \partial \Omega$ such that $\left|\partial_{t} u\left(X^{j}\right)\right| \geq c>0$, then one may scale at $X^{j}$ with $d_{j}=\operatorname{dist}\left(X^{j}, \partial \Omega\right)$ and define $u_{j}(X):=\left[u\left(d_{j} x+x^{j}, d_{j}^{2} t+t^{j}\right)-u\left(X^{j}\right)\right] / d_{j}^{2}$ to end up with a global solution $u_{\infty}$ with the property $\partial_{t} u_{\infty}(0) \neq 0$, contradicting Proposition 3.2 ,

The proof of the following result is a minor modification of the one of [10, Lemma 4.1], so we just give a sketch of the proof.

Lemma 4.2. Let $u: Q_{1} \rightarrow \mathbb{R}$ be $a W_{x}^{2, n} \cap W_{t}^{1, n}$ solution of (1.1) with $\Omega \supset\{u \neq 0\}$. Assume that for some space-time direction $e=\left(e_{x}, e_{t}\right)$ with $|e|=1$ we have $C_{0} \partial_{e} u-u \geq-\varepsilon_{0}$ in $Q_{1}$ for some $C_{0}, \varepsilon_{0} \geq 0$, and that $F$ is convex and satisfies (H0)-(H1). Then $C_{0} \partial_{e} u-u \geq 0$ in $Q_{1 / 2}$ provided $\varepsilon_{0} \leq \frac{1}{4\left(2 n \lambda_{1}+1\right)}$.

Proof. Since $F$ is convex, for any matrix $M$ we can choose an element $P^{M}$ inside $\partial F(M)$ (the subdifferential of $F$ at $M$ ) in such a way that the map $M \mapsto P^{M}$ is measurable, and we define the measurable uniformly elliptic coefficients

$$
a_{i j}(x, t):=\left(P^{D^{2} u(x, t)}\right)_{i j} \in \partial F\left(D^{2} u(x, t)\right) .
$$

As in the proof of [10, Lemma 4.1], by the convexity of $F$ if follows that, in the viscosity sense,

$$
a_{i j} \partial_{i j}\left(\partial_{e} u\right)-\partial_{t}\left(\partial_{e} u\right) \leq 0 \quad \text { in } \Omega
$$

and

$$
a_{i j} \partial_{i j} u-\partial_{t} u \geq 1 \quad \text { in } \Omega \text {. }
$$

Now, let us assume by contradiction that there exists $X^{0}=\left(x^{0}, t^{0}\right) \in Q_{1 / 2}$ such that $C_{0} \partial_{e} u\left(X^{0}\right)-u\left(X^{0}\right)<0$, and consider the function

$$
w(X):=C_{0} \partial_{e} u(X)-u(X)+\frac{\left|x-x^{0}\right|^{2}-\left(t-t_{0}\right)}{2 n \lambda_{1}+1} .
$$


Thanks to (4.1), (4.2), and assumption (H1) (which implies that $\lambda_{0} \mathrm{Id} \leq a_{i j} \leq \lambda_{1}$ Id) we deduce that $w$ is a supersolution of the linear operator $\mathscr{L}:=a_{i j} \partial_{i j}-\partial_{t}$, hence, by the minimum principle,

$$
\min _{\partial_{p}\left(\Omega \cap Q_{1}\left(Y^{0}\right)\right)} w=\min _{\Omega \cap Q_{1}\left(Y^{0}\right)} w \leq w\left(Y^{0}\right)<0 .
$$

By Lemma 4.1 and the assumption $\Omega \supset\{u \neq 0\}$ we have $\partial_{t} u=u=|\nabla u|=0$ on $\partial \Omega$, therefore $w \geq 0$ on $\partial \Omega$. Thus, since $\left|x-x^{0}\right|^{2}-\left(t-t^{0}\right) \geq 1 / 4$ on $\partial_{p} Q_{1}^{-}$it follows that

$$
0>\min _{\partial_{p} Q_{1 / 2}^{-}\left(X^{0}\right)} w \geq-\varepsilon_{0}+\frac{1}{4\left(2 n \lambda_{1}+1\right)},
$$

a contradiction if $\varepsilon_{0} \leq \frac{1}{4\left(2 n \lambda_{1}+1\right)}$.

\section{Proof of Theorem 1.2}

The proof of this theorem is very similar to the proof of [10, Theorem 1.3]. Indeed, take $X^{0}=\left(x^{0}, t^{0}\right) \in \partial \Omega \cap Q_{1 / 8}$, and rescale the solution around $X^{0}$, that is $u_{r}(x, t):=[u(r x+$ $\left.\left.x^{0}, r^{2} t+t^{0}\right)-u\left(x^{0}, t^{0}\right)-r \nabla u\left(x^{0}, t^{0}\right) \cdot x\right] / r^{2}$.

Because of the uniform $C_{x}^{1,1} \cap C_{t}^{0,1}$ estimate provided by Theorem 1.1 and the thickness assumption on the free boundary of $u$, we can find a sequence $r_{j} \rightarrow 0$ such that $u_{r_{j}}$ converges locally uniformly to a global solution $u_{\infty}$ of the form $u_{\infty}(x)=\gamma\left[\left(x \cdot e_{X^{0}}\right)_{+}\right]^{2} / 2$ with $\gamma \in\left[1 / \lambda_{1}, 1 / \lambda_{0}\right]$ and $e_{X 0} \in \mathbb{S}^{n-1}$ (see Proposition [3.2).

Notice now that, for any $s \in(0,1)$, we can find a large constant $C_{s}$ such that

$$
C_{s} \partial_{e} u_{\infty}-u_{\infty} \geq 0 \quad \text { inside } B_{1}
$$

for all directions $e=\left(e_{x}, e_{t}\right) \in \mathbb{S}^{n}$ such that $e \cdot\left(e_{X^{0}}, 0\right) \geq s$, hence by the $C_{x}^{1}$ convergence of $u_{r_{j}}$ to $u_{\infty}$ and Lemmas 4.1 and 4.2 we deduce that

$$
C_{s} \partial_{e} u_{r_{j}}-u_{r_{j}} \geq 0 \quad \text { in } Q_{1 / 2},
$$

and since $u_{r_{j}}(0)=0$ a simple ODE argument shows that $u_{r_{j}} \geq 0$ in $Q_{1 / 4}$.

Using (5.1) again, this implies that $\partial_{e} u_{r_{j}}$ inside $Q_{1 / 4}$, and so in terms of $u$ we deduce that there exists $r=r(s)>0$ such that

$$
\partial_{e} u \geq 0 \quad \text { inside } Q_{r}\left(X^{0}\right)
$$

for all $e \in \mathbb{S}^{n}$ such that $e \cdot\left(e_{X^{0}}, 0\right) \geq s$.

A simple compactness argument shows that $r$ is independent of the point $x$, which implies that the free boundary is $s$-Lipschitz. Since $s$ can be taken arbitrarily small (provided one reduces the size of $r$ ), this actually proves that the free boundary is $C^{1}$. Higher regularity is then classical.

\section{Appendix: Parabolic BMO estimates}

Let $u: Q_{1} \rightarrow \mathbb{R}$ satisfy $|u| \leq 1$ and $|\mathcal{H}(u)| \leq M$. Up to replacing $u$ by $u\left(x / R, t / R^{2}\right)$ and $\mathcal{H}$ by $\left(F\left(R^{2} \cdot\right) / R^{2}-\partial_{t}\right)$ with $R$ a large fixed constant, we can assume that $|\mathcal{H}(u)| \leq \delta$ with $\delta$ a small constant to be fixed later. Observe that, with this scaling, the ellipticity remains the same.

Let us first state a standard stability result. 
Lemma 6.1. (Compactness) Let $\varepsilon>0$, and $u$ be such that $u: Q_{1} \rightarrow \mathbb{R}$ satisfy $|u| \leq 1$. Let further $v: Q_{1 / 2} \rightarrow \mathbb{R}$ solve

$$
\begin{cases}\mathcal{H}(v)=0 & \text { in } Q_{1 / 2} \\ v=u & \text { on } \partial Q_{1 / 2}\end{cases}
$$

Then there exists $\delta=\delta(\varepsilon)>0$ such that

$$
|u-v| \leq \varepsilon \quad \text { in } Q_{1 / 2}
$$

provided $|\mathcal{H}(u)| \leq \delta$.

The proof of the lemma is based on a standard compactness argument, using that both $u$ and $v$ are uniformly Hölder continuous (in $(x, t)$-variables) inside $Q_{1 / 2}$; see [12], Lemma 5.1.

We say that $P$ is a "parabolic" second order polynomial if it is of the form

$$
P(x, t)=a_{0}+\left\langle b_{0}, x\right\rangle+\left\langle M_{0} x, x\right\rangle+c_{0} t, \quad a_{0}, c_{0} \in \mathbb{R}, b_{0} \in \mathbb{R}^{n}, M_{0} \in \mathbb{R}^{n \times n} .
$$

We now prove by induction the following result:

Lemma 6.2. Let $u: Q_{1} \rightarrow \mathbb{R}$ be a solution to our problem (1.1), with $|u| \leq 1$. Ten there exists $\rho>0$ universal such that

$$
\left|u(X)-P_{k}(X)\right| \leq \rho^{2 k} \quad \text { inside } Q_{\rho^{k}} \quad \forall k \in \mathbb{N},
$$

where $P_{k}$ is a parabolic second order polynomial such that $\mathcal{H}\left(P_{k}\right)=0$.

A straight forward implication of this result is that there is a universal constant $C=1 / \rho^{2}$ such that

$$
\left|u(X)-P_{r}(X)\right| \leq C r^{2} \quad \text { inside } Q_{r} \quad \forall 0<r<1,
$$

where $P_{r}$ is a parabolic second order polynomial such that $\mathcal{H}\left(P_{r}\right)=0$. This in turn implies an $L^{p}$-BMO type result, see the corollary below.

Proof. (of Lemma 6.2) Since the result is obviously true for $k=0$ (just take $P_{0}=0$ ), we prove the inductive step. So, let us assume that the result is true for $k$ and we prove it for $k+1$.

Define $u_{k}(X):=\frac{u\left(\rho^{k} x, \rho^{2 k} t\right)-P_{k}\left(\rho^{k} x, \rho^{2 k} t\right)}{\rho^{2 k}}$. Then, by the inductive hypothesis $\left|u_{k}\right| \leq 1$ inside $Q_{1}$. In addition

$$
\left|\mathcal{H}_{k}\left(u_{k}\right)\right| \leq \delta, \quad \mathcal{H}_{k}(v):=F\left(D^{2} v+D^{2} P_{k}\right)-\partial_{t} P_{k}-\partial_{t} v
$$

Observe that $\mathcal{H}_{k}$ keeps the same ellipticity as $\mathcal{H}$. Hence we can apply the lemma above to deduce that

where $v_{k}$ solves

$$
\left|u_{k}-v_{k}\right| \leq \varepsilon \quad \text { in } Q_{1 / 2},
$$

$$
\begin{cases}\mathcal{H}_{k}\left(v_{k}\right)=0 & \text { in } Q_{1 / 2} \\ v_{k}=u_{k} & \text { on } \partial Q_{1 / 2}\end{cases}
$$

Since $\left\|v_{k}\right\|_{L^{\infty}\left(Q_{1 / 2}\right)} \leq\left\|u_{k}\right\|_{L^{\infty}\left(Q_{1}\right)} \leq 1$, by interior $C_{\alpha}^{2,1}$ estimates we get

$$
\left\|v_{k}\right\|_{C_{\alpha}^{2,1}\left(Q_{1 / 4}\right)} \leq C_{0} .
$$

Let $\hat{P}_{k}$ be the "parabolic" second order Taylor expansion of $v_{k}$ at $(0,0)$, and notice that $\mathcal{H}_{k}\left(\hat{P}_{k}\right)=$ $\mathcal{H}_{k}\left(v_{k}(0,0)\right)=0$. Then

$$
\left|v_{k}-\hat{P}_{k}\right| \leq C_{0} \rho^{2+\alpha} \quad \text { inside } Q_{\rho}
$$


which gives

$$
\left|u_{k}-\hat{P}_{k}\right| \leq C_{0} \rho^{2+\alpha}+\varepsilon \quad \text { inside } Q_{\rho} .
$$

In particular, if we choose $\rho$ sufficiently small so that $C_{0} \rho^{\alpha} \leq 1 / 2$ and then $\varepsilon \leq \rho^{2} / 2$ we arrive at

$$
\left|u_{k}(X)-\hat{P}_{k}(X)\right| \leq \rho^{2} \quad \text { inside } Q_{\rho},
$$

or equivalently (recalling the definition of $u_{k}$ )

$$
\left|u(X)-P_{k+1}(X)\right| \leq \rho^{2(k+1)}, \quad P_{k+1}(X):=P_{k}(X)+\rho^{2 k} \hat{P}_{k}\left(x / \rho^{k}, t / \rho^{2 k}\right) .
$$

Also, since $\mathcal{H}_{k}\left(\hat{P}_{k}\right)=0$ we will have

$$
\mathcal{H}\left(P_{k+1}\right)=F\left(D^{2} P_{k+1}\right)-\partial_{t} P_{k+1}=F\left(D^{2} P_{k}+D^{2} \hat{P}_{k}\right)-\partial_{t} P_{k}-\partial_{t} \hat{P}_{k}=\mathcal{H}_{k}\left(\hat{P}_{k}\right)=0
$$

which concludes the proof of the inductive step.

Remark 6.3. As a corollary of our result we deduce $L^{p}$-BMO estimates on $\tilde{D}^{2} u(p \in(1, \infty))$ for solutions to general elliptic/parabolic operators of type $F=F\left(\tilde{D}^{2} u, \nabla u, u, X\right)$ provided $F$ is Hölder continuous and $u \in C_{x}^{1, \alpha}$.

Indeed, if $F=F\left(D^{2} u, X\right)$, since $u_{k}(X):=\frac{u\left(\rho^{k} x, \rho^{2} t\right)-P_{k}\left(\rho^{k} x, \rho^{2} t\right)}{\rho^{2 k}}$ satisfies $\left|u_{k}\right| \leq 1$ and $\left|\mathcal{H}_{k}\left(u_{k}\right)\right| \leq$ $\delta$ inside $Q_{1}$, by interior $W_{p}^{2,1}$ estimates we get

$$
\left\|\tilde{D}^{2} u_{k}\right\|_{L^{p}\left(Q_{1 / 2}\right)} \leq C,
$$

that is

$$
\frac{1}{\left|Q_{\rho^{k} / 2}\right|} \int_{Q_{\rho^{k} / 2}}\left|\tilde{D}^{2} u-\tilde{D}^{2} P_{k}\right|^{p} \leq C \quad \forall k \in \mathbb{N} .
$$

For general operators $F$ it suffices to apply the above argument to $G(M, X):=F(M, D u(X), u(X), X)$.

\section{REFERENCES}

[1] Andersson J.; Lindgren E.; Shahgholian H.; Optimal regularity for the no-sign obstacle problem. Comm. Pure. Appl. Math., 66 (2013), no. 2, 245- 262.

[2] Andersson J.; Lindgren E.; Shahgholian H.; Optimal regularity for parabolic no-sign obstacle problem. Preprint.

[3] Caffarelli, L. A.; Interior a priori estimates for solutions of fully nonlinear equations. Ann. of Math. (2) 130 (1989), no. 1, 189-213.

[4] Caffarelli, L. A.; Huang, Q.; Estimates in the generalized Campanato-John-Nirenberg spaces for fully nonlinear elliptic equations. Duke Math. J. 118 (2003), no. 1, 1-17.

[5] Caffarelli, L. A.; Karp L.; Shahgholian, H.; Regularity of a free boundary with application to the Pompeiu problem. Ann. of Math. (2), 151 (2000), no. 1, 269-292.

[6] Caffarelli, L. A.; Petrosyan, A.; Shahgholian, H.; Regularity of a free boundary in parabolic potential theory. J. Amer. Math. Soc., 17 (2004), no. 4, 827-869.

[7] Crandall, M. G.; Kocan, M.; Święch, A.; $L^{p}$-theory for fully nonlinear uniformly parabolic equations. Comm. Partial Differential Equations, 25 (2000), no. 11-12, 1997âĂŞ2053.

[8] Friedman, A.; Variational principles and free-boundary problems. A Wiley-Interscience Publication. Pure and Applied Mathematics. John Wiley \& Sons, Inc., New York, 1982.

[9] Krylov, N. V.; Boundedly inhomogeneous elliptic and parabolic equations. (Russian) Izv. Akad. Nauk SSSR Ser. Mat., 46 (1982), no. 3, 487-523

[10] Figalli, A.; Shahgholian, H. ; A free boundary problem for fully nonlinear elliptic equations. Preprint. 
[11] Petrosyan, A.; Shahgholian, H.; Uraltseva, N., Regularity of free boundaries in obstacle-type problems. Graduate Studies in Mathematics, 136. American Mathematical Society, Providence, RI, 2012.

[12] L. Wang, On the regularity theory of fully nonlinear parabolic equations. I. Comm. Pure Appl. Math., 45 (1992), no. 2, 27-76.

[13] L. Wang, On the regularity theory of fully nonlinear parabolic equations. II. Comm. Pure Appl. Math., 45 (1992), no. 2, 141-178.

Mathematics Department, The University of Texas at Austin, Austin, Texas, 78712-1202, USA E-mail address: figalli@math.utexas.edu

Department of Mathematics, KTH Royal Institute of Technology, 10044 Stockholm, Sweden E-mail address: henriksh@kth.se 